\title{
Dynamic Vulnerability Analysis of Population for Flood Disaster in Urban Area
}

\author{
Xilei Pang \\ Emergency Management Department, Dalian Adminstrative College \\ Dalian 116013, China, pxlbbang@163.com \\ Received 1 October 2013 \\ Accepted 12 December 2013
}

\begin{abstract}
Vulnerability analysis is a key step of risk analysis of natural disaster. Based on information diffusion theory and incomplete information, it analyzes the changes of the affected population in urban flood and identifies the function relationship between the hazard (rainstorm) and disaster-bearing body (population), which is called vulnerability curve of population in flood disaster.This curve not only can present some changing extent of urban flood risk, but also could provide an important basis for integrated risk assessment of urban flood.
\end{abstract}

Keywords: Vulnerability, Flood, Disaster-bearing Body, Population, Dynamic

\section{城市洪涝灾害的人口脆弱性动态分析}

\author{
庞西嗄 \\ 大连行政学院应急管理教研部，大连，116013
}

摘 要: 承灾体脆弱性分析是自然灾害风险分析的关键步骤之一。基于信息扩散技术, 本文利用小样本数据, 在框定时间约束的前提下, 较客观地分析了城市洪涝灾害系统中单一承灾体（受灾人口）的动态变化情况, 并识别和近似推理出致灾因子 (暴雨) 与承灾体 (人口) 之间的函数关系, 即人口脆弱性变化曲线。该曲线 在一定程度上可以反映出研究案例区的洪涝灾害风险发展变化情况, 并为自然灾害的综合风险评估提供重要 依据。

关键词：脆弱性，洪涝灾害，承灾体，人口，动态

\section{1 研究背景}

有史以来, 洪涝灾害一直是许多国家和地区面 临的主要自然灾害之一。由于全球变化引起的区域 气候波动，导致极端暴雨天气的频繁出现，洪涝灾 害的发生频次已经超过台风、地震、泥石流、干旱 等自然灾害 (参见图1), 逐渐成为危害城市安全和 社会、经济发展的头号公敌。有权威统计报告 ${ }^{[1]}$ 显示, 中国近十年来的洪涝灾害造成的受灾人口和经济损 失规模均列世界首位, 而这些洪涝灾害有很大一部 份都发生在人口集中和经济发展迅速的城镇地区。

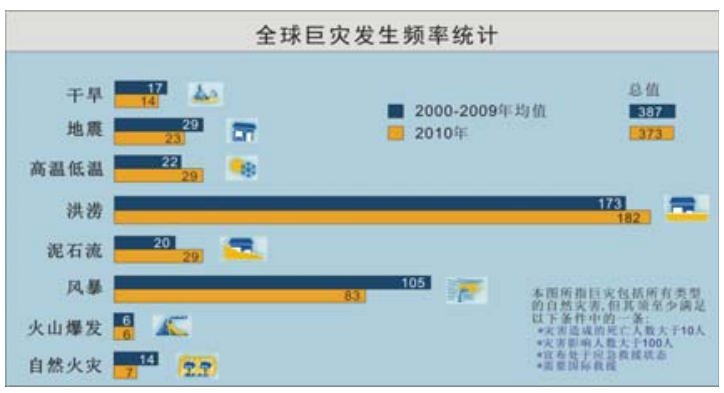

图1２1世纪初全球巨灾发生频率对比图
长期以来, 国内的城市洪涝灾害风险研究主要集中 在暴雨降水预测、洪水动力模型、致灾因子危险性 分析等方面, 这些研究重在计算和分析灾害发生的 可能性方面, 多是利用暴雨洪涝形成的物理数学模 型和计算机仿真模拟技术，从不同侧面对城市洪涝 灾害风险进行了不同层次的探讨和研究。

随着认知水平的提高, 人类对自然灾害的本质 和演变规律的认识也发生着变化。即便是在相当长 一段时间内某种自然灾害的演变规律是不变的, 但 时刻都会发展变化的社会经济系统, 也会导致区域 自然灾害风险发生明显的变化。自然灾害的风险水 平, 主要与致灾因子的强弱和人类社会的脆弱性有 关。脆弱性是系统暴露在因气候变化引起的灾害事 件之后调节风险变化的因素 ${ }^{[2]}$, 当致灾因子的破坏性 强且社会系统的脆弱性也高时, 自然灾害的风险水 平就高。从时间尺度上看, 脆弱性研究不仅要关注 长时间内全球气候变化的缓慢过程, 也关注短期内 突发性环境事件的影响, 脆弱性会在较短时间内从 极其脆弱突变为极其安全 ${ }^{[3]}$ 。从系统学角度看, 社会 -经济-生态系统的脆弱性、恢复力和适应能力三者之 间是相互影响和制约的 ${ }^{[4]}$ 。

Published by Atlantis Press

Copyright: the authors 
Xilei Pang

当前, 自然灾害领域的脆弱性研究越来越关注 自然系统与社会系统之间的复杂作用关系，其未来 的发展越来越取决于多学科研究方法、研究视角的 交叉以及多源数据多学科知识的融合。

关于脆弱性研究, 有人关注于概念和框架的理 解, 也有人热衷于运用加权处理、层次分析等方法 进行脆弱性评价。例如, 有研究重在用多指标体系 建立脆弱性分析模型 ${ }^{[5-6]}$; 也有研究从自然灾害系统 入手进行机理分析的探索，并得出“脆弱性是区域灾 害系统中致灾因子、承灾体和孕灾环境综合作用过 程的状态量, 它主要取决于区域的经济发达程度与 社区安全建设水平”的结论 ${ }^{[7]}$ 。有研究认为, 经济的 发展使我们进入“系统的系统”时代，许多关键设施 面临高风险, 社会经济脆弱性并没有降低 ${ }^{[8]}$ 。也有研 究人为: 随着收入的增加, 灾害的死亡人数也在增 加, 一旦超过某个拐点, 灾害死亡人数就开始下降, 因此, 转变经济发展方式, 实现“包容性增长”, 才 是降低社会经济脆弱性的根本 ${ }^{[9]}$ 。虽然人们发现灾害 损失和 GDP 之间存在着经验关系 ${ }^{[10]}$, 但人口密度等 数据在研究生命易损性时也很重要 ${ }^{[11]}$ 。理论上讲, 经济社会发展评价指标体系越完善, 脆弱性的评价 就越可靠, 但从成本角度看, 有限的几个主要指标 就可以支撑脆弱性分析。

脆弱性的形成原因及表现特征在空间上具有很 强的区域差异性, 在时间上具有动态变化性。因此, 要想跨区域、跨时段、跨学科地建立科学合理的脆 弱性评价指标体系是非常困难的。此外，指标体系 评价法所得出的评价结果的有效性也很少被验证。

因此，仅仅依靠指标值计算和权重赋值而得到的脆 弱性分析结果，会显得有些单薄，而且权重的大小 从某种角度讲过于主观。

自然灾害是自然和人类社会相互作用的复杂 现象。从系统论角度看, 灾害风险是由风险源、风 险载体和人类社会的防减灾措施等三方面因素相 互作用而形成的 ${ }^{[12]}$; 自然灾害风险研究应该从孕灾 环境、致灾因子、承灾体、承灾能力、灾害损失等 多项构成自然灾害系统的基本要素着手研究 ${ }^{[13]}$ 。

由上可知, 承灾体脆弱性研究是自然灾害风险 分析不可或缺的关键步骤。因此, 为了解决人们在 日常研究中经常碰到的数据缺乏、过分依赖主观赋 分评价和难以准确量化多因素关系的现状，本文基 于综合风险评估的基本模式 ${ }^{[14]}$, 在尽量避免主观评 价的前提下, 利用信息扩散技术, 以城市洪涝灾害 为例, 开展单一指标的脆弱性分析研究。

\section{2 承灾体脆弱性分析}

\section{1 承灾体脆弱性的动态分析模式}

进行承灾体脆弱性分析时, 需要考虑承灾体的 “时”、“空”、“度”等因素。其中，“时”指承灾体遭受 灾害影响的时间，此时间可以是时间点，也可以是 时间段; “空”指承灾体所处的空间地理位置; “度” 指承灾体的受影响程度，比如财产的损失量、建筑 物的倒塌数量和破损程度、人员伤亡的数量等等。
如果用 $V I$ 表示承灾体脆弱性指标, 用 $h$ 代表致 灾因子变量, $T$ 为承灾体变化的时间变量。则, 承灾 体脆弱性动态评估的基本模式可以用以下示意图表 达（见图 2)。

承灾体一般是指社会经济系统中的经济、人口、 居民地、建筑物、土地、道路、机场、军用设施以 及地下管线等公共基础设施在内的，一切与人类活 动有关的客观存在。人们一般会用城市形态结构、 人口结构、基础设施结构，或是人口密度、人口年 龄结构、经济密度、建筑物密度、生命线工程密度、 道路敏感度等作为承灾体脆弱性的评价指标 ${ }^{[15-16]}$ 。

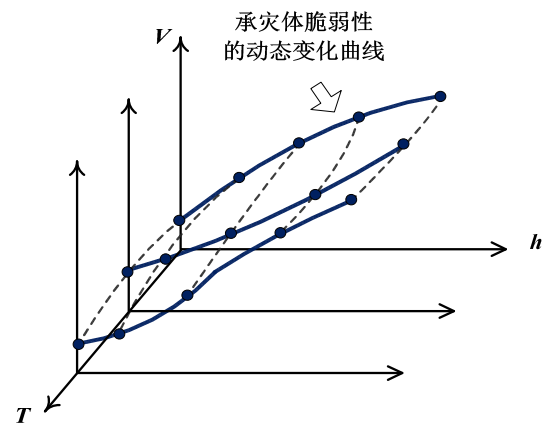

图2 承灾体脆弱性动态评估的三维示意图

承灾体的脆弱性直接反映了社会经济系统中不 同层次的承灾体遭遇过或有可能在未来遭遇灾害时 所表现的某种性质, 但是由于不同承灾体的度量单 位不一样, 往往会造成脆弱性的评估量纲难以统一 标准化。因此, 本文建议用经济损失率、受影响人 口比例等表示承灾体的脆弱性指标, 即灾情与相应 社会经济发展指标的比值。美国保险业就是用受淹 水深增加时不同类型建筑的损失率来表达建筑物的 洪水脆弱性 ${ }^{[17]}$ 。如果我们用 $D$ 表示灾情, $S$ 表示社 会经济发展指标, 用 $V$ 表示承灾体的脆弱性指标, 则有: $V=D / S$ 。

\section{2 脆弱性曲线}

承灾体脆弱性曲线不是一条主观曲线, 而是要 通过大量因果关系研究给出的。评估脆弱性的一种 基本方法是：用同一发展时期的灾害数据统计出一 条脆弱性曲线, 再由多个发展时期的多条脆弱性曲 线统计出曲线族的变化规律, 然后引入庞加莱-契达 耶夫方程, 建立社会发展的广义力学系统运动微分 方程，在相关边界条件下求得理论意义上的脆弱性 曲线, 最后再用理论意义上的脆弱性曲线去修订统 计意义上的脆弱性曲线 ${ }^{[18-19]}$ 。然而, 这种方法需要 大量的多领域的历史统计资料，同时建立一系列的 广义力学系统运动微分方程也相当复杂, 目前只是 在理论构想阶段, 尚没有具体算法和模型出现。

鉴于上述情况，人们利用相对简便易懂的剂量反应函数（Dose Response Functions）来表示致灾因 子强度与受灾程度之间的关系, 其几何表达称为剂 量-反应曲线（见图 3)。

Published by Atlantis Press

Copyright: the authors 


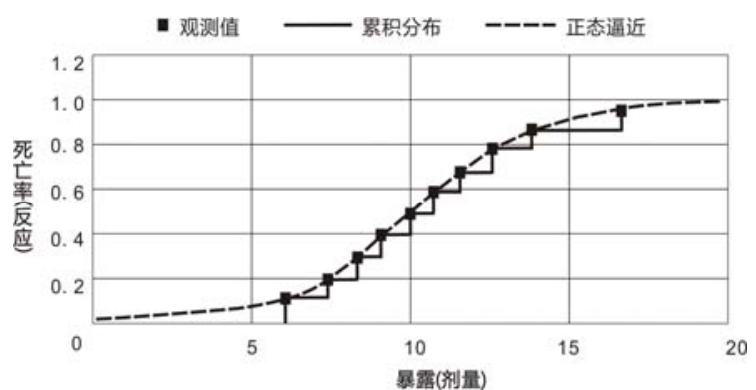

图 3 “暴露-死亡率” 的剂量-反应曲线示意图

剂量-反应原本是用来表达剂量和不良健康之 间数学关系的一种函数 ${ }^{[20-21]}$ 。美国科学院国家研究 委员会在 1983 年的“联邦政府的风险评估报告”中, 正式将其用于一般性的风险评估中。

剂量-反应函数的特征表现形式主要有三种，分 别是离散剂量-反应函数、指数分布剂量-反应函数和 概率分布剂量-反应函数。

(1) 离散的剂量-反应函数

最简单的剂量-反应函数的特征形式是离散的 剂量-反应函数。关于溃坝洪水灾害分析 ${ }^{[22]}$ 和地震灾 害中的房屋倒塌破坏性分析 ${ }^{[23]}$ 等相关研究, 就是利 用多情景的离散剂量-反应曲线表达不同承灾体的 脆弱性。

（2）指数分布剂量-反应函数

很多情况下, 人们是得不到足够的数据完全反 映 $[0,1]$ 之间的剂量-反应函数关系的, “反应”也不一 定都是当“剂量”达到某一临界值才会发生变化的, 一些剂量-反应的变化过程是曲线的、循序渐进的。 于是, 连续型的剂量-反应函数更多地得以应用。例 如, 有研究利用正态分布函数计算人在流水中不稳 定性的不确定性概率问题 ${ }^{[24]}$; 也有研究用历史数据 的实证统计分析，指出了洪水水深与死亡率之间的 相关性 ${ }^{[25]}$, 可以用上升连续的指数分布函数 (曲线) 描述。

（3）概率分布剂量-反应函数

概率分布剂量-反应函数是最常用的剂量-反应 函数, 也称之为对数正态分布剂量-反应函数 ${ }^{[26]}$ 。该 模型认为“剂量”和 “反应”之间的关系可以用累计正 态分布表示（见图 4)。这里, 概率可以用来描述不 同环境条件下的致命或非致命健康影响。概率分布 剂量-反应函数常用来表示人类在爆炸、毒气扩散、 热辐射等环境下的“反应”情况 ${ }^{[27]}$ 。

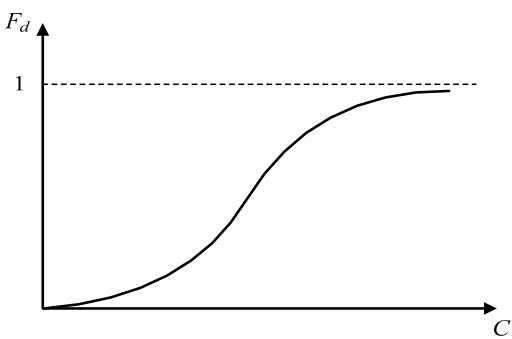

图 4 概率分布/对数正态分布剂量-反应函数
一般的, 剂量-反应函数的研究建立在大量的真 实物理实验之上, 但是在一些领域（比如自然灾害 领域的人员伤亡和建筑物损毁), 不管是从伦理上还 是成本角度上看, 进行大量的物理实验显然是不可 能的。因此, 我们获得相关数据的途径只能是收集 历史统计数据或开展仿真模拟实验。

\section{3 信息扩散理论}

从某种意义上讲, 人们掌握的用于进行风险分 析的信息往往是不完备的, 如果人们能获得足以认 清未来的信息时, 风险也就不存在了。针对自然灾 害风险分析领域经常遇到不完备信息的这一情况, 有学者经过长期的试验、验证以及多领域的应用研 究 ${ }^{[28-31]}$, 系统地提出了一种处理不完备信息的理论 方法，即信息扩散技术。

信息扩散技术是一种模糊分析技术，它在不假 设样本属于任何分布的前提下, 可以尽可能多地挖 掘出数据资料本身所蕴藏的有用信息，提高系统识 别的精度, 其解决了从普通样本转变为模糊样本的 问题，从而超越了传统模糊集技术依赖专家选定隶 属函数的随意性, 在一定程度上可以保证分析结果 的客观性。该技术可以有效地利用有限信息，较合 理地分析系统中单个因素的变化规律, 并可以识别 和近似推理出系统因素间的函数关系 (见图 5)。

基于信息矩阵法的函数关系识别和计算过程， 可以归纳为构造信息矩阵、生成模糊关系矩阵和近 似推理三个步骤。

\section{步骤 1 : 构造信息矩阵}

首先, 需要准备样本 $G$, 如果 $G$ 中含有 $n$ 个样 本点 ( $n$ 也称为 $G$ 的样本容量), 每个样本点有两个 分量, 这两个分量分别是输入 $I$ 和输出 $O$ 。则该样本 可记为: $G=\left\{\left(I_{1}, O_{1}\right),\left(I_{2}, O_{2}\right), \ldots,\left(I_{n}, O_{n}\right)\right\}$

然后，制定样本 $I$ 和 $O$ 对应的监控空间 $U=\left\{u_{1}, u_{2}, \ldots, u_{s}\right\}$ 和 $V=\left\{v_{1}, v_{2}, \ldots, v_{t}\right\}$ 。监控空间 $U$ 和 $V$ 的大小应根据研究需要设定, 我们称相邻监控点 之间的距离为步长, 用 $\Delta$ 表示, $\Delta$ 可以是等长的, 也可以不等长。但原则上监控点的个数须小于样本 容量, 以便所有样本点的信息都能全部分配到监控 空间中。

最后，利用分明区间和模糊区间的信息矩阵以 及正态信息扩散扩散函数的计算公式 ${ }^{[32]}$, 计算每个 样本点在监控空间上的信息增量 $Q_{s t}$, 进而组成由 $U$ 和 $V$ “控制” 的信息矩阵 $I M$ 。

$$
I M=\begin{gathered}
v_{1} \\
u_{1} \\
u_{2} \\
\vdots \\
u_{s}
\end{gathered}\left(\begin{array}{cccc}
Q_{11} & Q_{12} & \cdots & Q_{t t} \\
Q_{21} & Q_{22} & \cdots & Q_{2 t} \\
\vdots & \vdots & \vdots & \vdots \\
Q_{s 1} & Q_{s 2} & \cdots & Q_{s t}
\end{array}\right)
$$

步骤 2 : 生成模糊关系矩阵 
自然灾害风险分析领域常常遇到小样本问题, 虽然可使用的样本点不多, 但不能因为难以统计出 其规律而放弃研究。样本点少, 同样会有一定的规 律，其提供的信息一般可以用来进行模糊关系分析。

假设我们通过小样本计算得到了信息矩阵 $I M$,

$U$ 和 $V$ 可以被看作是描述和控制 的两个向量, 我们 也可以称以这种形式出现的 $U$ 和 $V$ 为状态因素空 间, 因素空间就像是几何中的坐标系, 它提供了一 种用来描述事物状态的参考环境。当我们把 $U \times V$ 看成是一个因素空间时, $V$ 的元素则可被视为概念, $U$ 可被视为概念的论域, 而概念的形式大小则由信 息矩阵 $I M=\left\{Q_{j k}\right\}_{s \times t}$ 来限定。

令:

且:

$$
S_{k}=\max _{1 \leq j \leq s}\left\{Q_{j k}\right\}, \quad k=1,2, \ldots, t
$$

$$
\psi_{k}\left(u_{j}\right)=\frac{Q_{j k}}{S_{k}}, j=1,2, \ldots, s
$$

那么, 通过信息矩阵 $I M, V$ 中的任意一个元素 $v_{k}$ 都可以用由 $U$ 表示的一个模糊集 $\psi_{k}$ 来表示。如果 用 $r_{j k}$ 表示 $\psi_{k}\left(u_{j}\right) 、 F M$ 代表由所有 $\psi_{k}\left(u_{j}\right)$ 组成的矩 阵。则我们说通过信息矩阵 $I M$ 得到了一个关于 $U$ 和 $V$ 的因果模糊关系矩阵 $F M=\left\{r_{j k}\right\}_{s \times t}$ 。

\section{步骤 3: 近似推理}

当我们通过信息矩阵计算得到了一个因果模糊 关系矩阵后, 大体上相当于得到了一个函数关系 $y=f(x)$ 。然而, 模糊关系矩阵只能表达输入-输出的 近似关系, 那么就需要用模糊近似推理的办法, 将 模糊关系矩阵 $F M$ 用隶属函数的形式表达为 $Y=F(x)$ (见图 5)

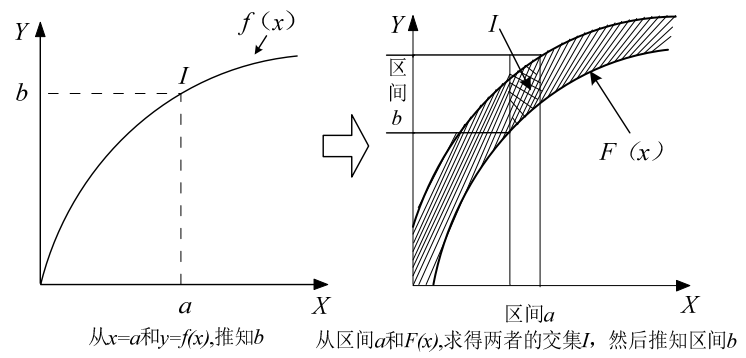

图 5 模糊近似推理的简单示意图

有关近似推理的原理和具体步骤, 请参阅相关 参考文献 ${ }^{[32]}$ 。

\section{3 城市洪涝灾害的人口脆弱性案例分析}

汕头市在赤道低气压带和副热带高气压带之 间, 属亚热带海洋性气候, 雨量丰沛, 但降雨量时 空和地域分布不均匀, 呈增多态势。按照国家气象 局的降雨强度 (24 小时降雨) 等级划分标准, 汕头 市历史上（1951 年 1 月 1 日 2010 年 12 月 31 日） 发生过暴雨 348 次、大暴雨 103 次、特大暴雨 4 次, 年均 7.6 次, 强降水频率逐年增多。在炎热多雨、地 面排水不畅和防灾减灾措施缺乏等情况下, 在经济
发达、人口密集和建筑物林立的区域就容易造成暴 雨洪涝灾害。

基于以上情况, 本研究选择暴雨内涝灾害频繁 发生的汕头市为案例区。

\section{1 技术路线与数据准备}

(1) 技术路线

基于收集整理的汕头市历年的人口及洪涝灾情 数据, 首先选取受灾人口比例为脆弱性指标; 然后 根据政府制定政策的时间规律，以 5 年为单位框定 时间尺度; 最后，利用信息扩散技术计算和分析汕 头市洪涝灾害的人口脆弱性动态变化情况。

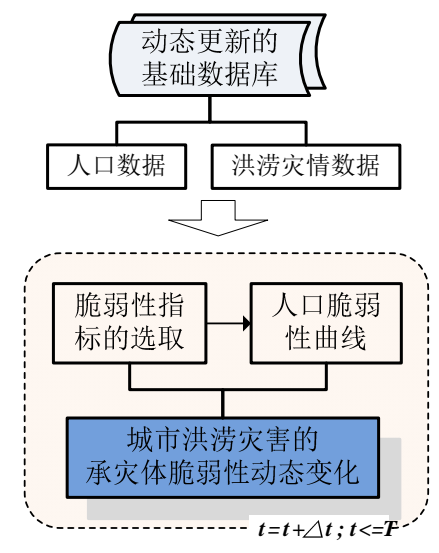

图 6 城市洪涝灾害的人口脆弱性分析流程

（2）时间约束

城市洪涝灾害的承灾体包括人口、建筑物、地 下管线、道路及其他基础设施等。承灾体的空间分 布、数量、大小都会随着时间的变化而发生变化。 大多数情况下, 人们会根据研究需要有针对性地选 取相应时间尺度的资料数据进行评估和分析。以人 口为例, 有统计意义的人口数据一般是以年为单位 存储的, 我国公共管理部门的社会、经济发展规划 又常常会以 5 年为单位进行更新。

结合承灾体可用数据的统计情况, 基于以年为 单位的人口、洪涝灾情等数据, 并根据研究需要, 从我国经济发展的“五年”计划角度出发, 对相关数 据进行分段分析, 最终分别以年和“五年计划”为单 位开展人口脆弱性的动态分析。

(3) 数据准备

用于承灾体脆弱性分析的数据主要包括研究 区的历年洪涝灾情数据和人口数据。汕头市历年的 洪涝灾害灾情数据（受灾人口）则是通过对汕头市 历年的防灾减灾年鉴、气象志、县志以及汕头市地 方各级政府的三防办、民政局、水利部门和农业局 等部门的统计资料进行收集整理得到。汕头市的人 口数据来自于汕头市历年统计年鉴。

Published by Atlantis Press

Copyright: the authors 


\section{2 承灾体脆弱性的动态分析}

\subsection{1 承灾体脆弱性指标的选取}

承灾体的脆弱性直接反映社会经济系统中不同 层次的承灾体在遭遇过或有可能将遭遇灾害是表现 的某种性质, 本研究采用受灾人口比例作为城市人 口的脆弱性指标, 即受灾人口与区域人口的比值。

表 1 汕头市暴雨洪涝灾害的人口脆弱性指标

\begin{tabular}{|c|c|c|c|}
\hline 时段 & 年份 & $\begin{array}{c}\text { 降雨量 } \\
\text { (单位: mm) }\end{array}$ & 脆弱性指标 \\
\hline \multirow{5}{*}{ 七五 } & 1986 & 146 & 0.002 \\
\hline & 1987 & 153 & 0.027 \\
\hline & 1988 & 185 & 0.011 \\
\hline & 1989 & 130 & 0.017 \\
\hline & 1990 & 198 & 0.025 \\
\hline \multirow{5}{*}{ 八五 } & 1991 & 119 & 0.01 \\
\hline & 1992 & 105 & 0.045 \\
\hline & 1993 & 141 & 0.019 \\
\hline & 1994 & 182 & 0.106 \\
\hline & 1995 & 152 & 0.024 \\
\hline \multirow{5}{*}{ 九五 } & 1996 & 117 & 0.021 \\
\hline & 1997 & 150 & 0.062 \\
\hline & 1998 & 103 & 0.03 \\
\hline & 1999 & 139 & 0.007 \\
\hline & 2000 & 80 & 0.033 \\
\hline \multirow{5}{*}{ 十五 } & 2001 & 113 & 0.032 \\
\hline & 2002 & 118 & 0.026 \\
\hline & 2003 & 131 & 0.021 \\
\hline & 2004 & 114 & 0.007 \\
\hline & 2005 & 128 & 0.055 \\
\hline \multirow{5}{*}{ 十一五 } & 2006 & 216 & 0.059 \\
\hline & 2007 & 101 & 0.036 \\
\hline & 2008 & 230 & 0.004 \\
\hline & 2009 & 81 & 0.039 \\
\hline & 2010 & 124 & 0.009 \\
\hline
\end{tabular}

\subsection{2 人口脆弱性曲线}

在分析人口脆弱性曲线的变化之前, 需要先制 定人口脆弱性指标样本和样本监控空间。针对 1986-2010 年的样本, 我们用 $P V$ 表示其人口脆弱性 指标样本, 用 $X$ 和 $Y$ 分别表示样本的两个分量。其 中, $X_{25}=\left[x_{1}, x_{2}, x_{3}, \ldots, x_{25}\right]$ 表示受灾人口比例, $Y_{25}=\left[y_{1}, y\right.$ $\left.2, y_{3}, \ldots, y_{25}\right]$ 表示降雨量。
用 $U_{24}=\left[u_{1}, u_{2}, u_{3}, \ldots, u_{25}\right]$ 表示 $X_{25}$ 的监控空间，用 $V_{24}=\left[v_{1}, v_{2}, v_{3}, \ldots, v_{25}\right]$ 表示 $Y_{25}$ 的监控空间, 监控空间 $U$ 的步长 $\Delta U=(\operatorname{Max} X-\operatorname{Min} X) / 15, V$ 的步长为: $\Delta V=$

$(\operatorname{Max} Y-\operatorname{Min} Y) / 15$ 。然后, 利用正态信息扩散法, 生成由 $U_{24}$ 和 $V_{24}$ 监控的信息矩阵为:

$$
\begin{aligned}
& \begin{array}{lllllll}
0.002 & 0.009 & 0.016 & \ldots & 0.093 & 0.100 & 0.107
\end{array} \\
& Q_{25}=\begin{array}{c}
80 \\
90 \\
100
\end{array}\left(\begin{array}{cccccccc}
0.260 & 0.530 & 0.980 & \ldots & 0.000 & 0.000 & 0.000 \\
\ldots & 1.600 & 2.480 & 3.310 & \ldots & 0.000 & 0.000 & 0.000 \\
210 & \ldots & \ldots & \ldots & \ldots & \ldots & \ldots & \ldots \\
220 & 0.610 & 0.790 & 0.860 & \ldots & 0.090 & 0.140 & 0.160 \\
230 & 0.740 & 0.790 & 0.680 & \ldots & 0.030 & 0.040 & 0.050 \\
0.800 & 0.790 & 0.570 & \ldots & 0.010 & 0.010 & 0.010
\end{array}\right)
\end{aligned}
$$

然后, 将信息矩阵 $Q_{25}$ 转化为模糊关系矩阵 $R_{25}$, 则“1986-2010”期间的降雨量与受灾人口比例的模糊 关系矩阵为:

$$
R_{25}=\begin{gathered}
0.002 \\
80 \\
90 \\
100
\end{gathered}\left(\begin{array}{cccccccc}
0.067 & 0.009 & 0.016 & \ldots & 0.093 & 0.100 & 0.107 \\
\ldots & 0.185 & 0.226 & 0.300 & \ldots & 0.000 & 0.000 & 0.000 \\
210 & 0.411 & 0.449 & 0.525 & \ldots & 0.000 & 0.000 & 0.000 \\
220 & \ldots & \ldots & \ldots & \ldots & \ldots & \ldots & \ldots \\
230 & 0.157 & 0.143 & 0.137 & \ldots & 0.220 & 0.206 & 0.203 \\
0.206 & 0.143 & 0.108 & \ldots & 0.073 & 0.059 & 0.063 \\
& 0.143 & 0.090 & \ldots & 0.024 & 0.015 & 0.013
\end{array}\right)
$$

事实上，当我们得到了降雨量与受灾人口比例 的模糊关系矩阵时，已经大致可以看出两者之间的 变化关系了(见图 7)。

图 7 中方格的色彩值从蓝色到红色，表示降雨 强度与受灾人口比例的关系由弱到强。由上图可以 看出, 受灾人口比例的变化并不是随着降雨量的升 高而增加的，也就是说降雨量和受灾人口比例之间 并不是呈明显的正比或反比线性关系。为了能够通 过有限的数据, 较清晰地表达致灾因子与承灾体之 间的关系, 也就是脆弱性的变化趋势, 我们需要将 相对模糊的关系矩阵转化为一维的向量, 并转化为 更直观的曲线形式予以表达。

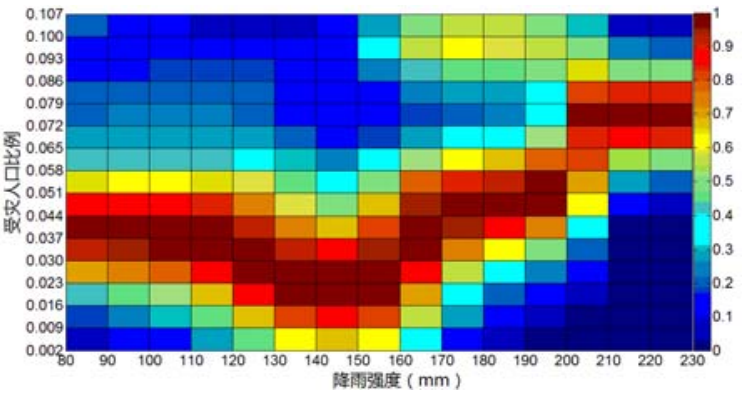

图 7 降雨强度与受灾人口比例的模糊关系色谱图

根据函数关系的模糊推理原理，对模糊关系矩 阵 $R_{25}$ 进行近似推理, 计算出 1986-2010 期间的人口 脆弱性变化向量 $L_{25}$ : 
$L_{25}=[0.041,0.039,0.038,0.037,0.038,0.041,0.045,0.051$, $0.060,0.070,0.075,0.074,0.069,0.064,0.060,0.055]$

$L_{25}$ 实质上是经过信息扩散和近似推理得到的 “受灾人口比例”在“降雨量”监控下的趋势向量, 这个 趋势向量就是人口脆弱性变化的数学表达, 其曲线 形式见图 8。

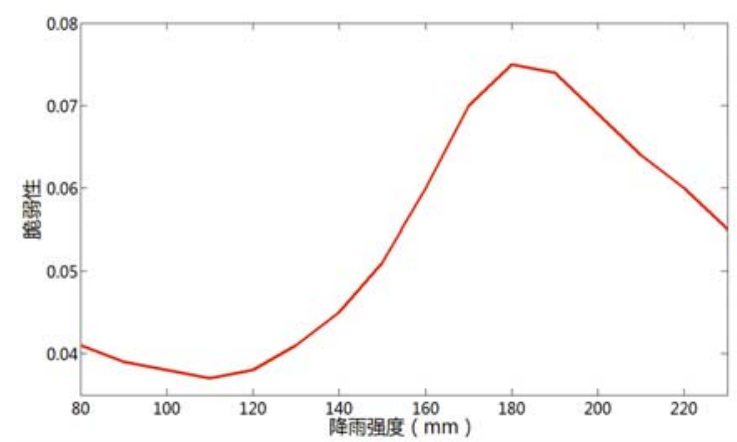

图 8 汕头市洪涝灾害人口脆弱性变化曲线

(1986-2010)

从图 8 中, 我们就可以看到受灾人口比例与降 雨强度之间的波动变化关系，但该曲线没有能够反 应出脆弱性曲线随着时间的推移而发生的动态变化 情况。因此，下面我们将 1986-2010 平均划分为 5 个阶段, 通过对 5 个阶段各自脆弱性的变化分析, 找出人口脆弱性在时间轴上的动态变化性。

按照上述计算方法，分别计算出 “七五”到“十 一五”各个阶段汕头市的降雨量与受灾人口比例之 间的模糊关系矩阵和人口脆弱性变化向量, 并得到 其变化曲线（见图 9-图 13）。

通过对 5 个“五年”计划期间的人口脆弱性分析， 我们分别得到了汕头市的“七五”、“八五”、“九五”、 “十五”和“十一五”期间的洪涝灾害人口脆弱性曲线, 这 5 条形状各异的脆弱性曲线, 不仅反映了每个五 年计划期间不同降雨强度条件下的人口脆弱性变化 情况，同时也指出了 5 个五年计划时段之间的脆弱 性变化也存在着很大的差异, 而且人口脆弱性的变 化并不是随着降雨强度的增加而线性增大或者线性 减小的。也就是说, 降雨和受灾人口之间存在着一 种复杂的模糊关系, 通过对已有的不完备信息进行 推理计算, 就可以得到一些离散的洪涝灾害人口脆 弱性值（见表 8), 将其连接, 便得到了近似意义上 的脆弱性曲线。

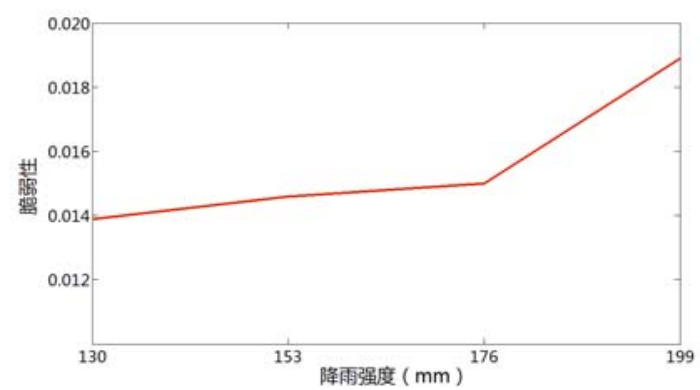

图 9 “七五” 期间汕头市洪涝灾害人口脆弱性曲线

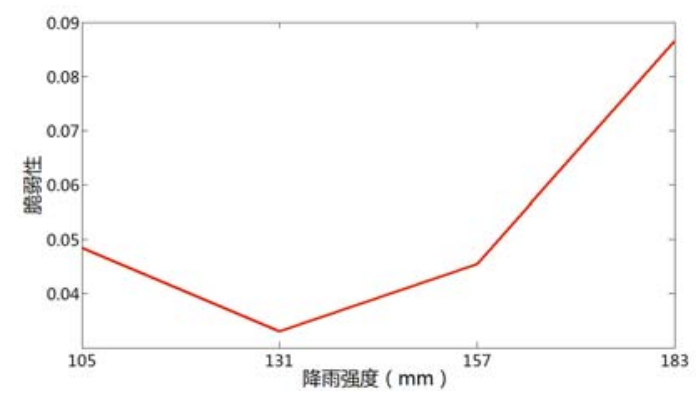

图 10 “八五” 期间汕头市洪涝灾害人口脆弱性曲线

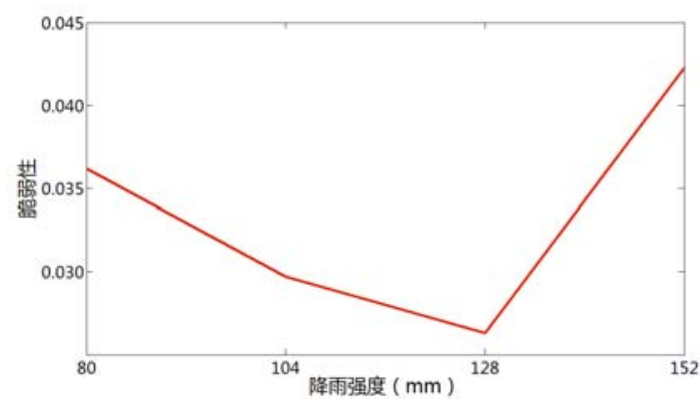

图 11 “九五” 期间汕头市洪涝灾害人口脆弱性曲线

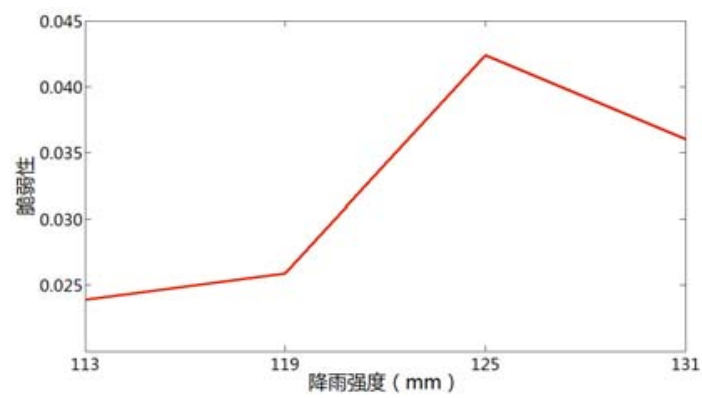

图 12 “十五” 期间汕头市洪涝灾害人口脆弱性曲线

表 8 汕头市洪涝灾害人口脆弱性动态变化值

\begin{tabular}{c|cccc|c|ccccc}
\hline 降雨均值 & \multicolumn{3}{|c|}{ 降雨强度 $(\mathrm{mm})$} & 时间段 & \multicolumn{3}{|c|}{ 脆弱性值 } & & 脆弱性均值 \\
\hline $165 \mathrm{~mm}$ & 130 & 153 & 176 & 199 & $1986-1990$ & 0.014 & 0.015 & 0.015 & 0.019 & 0.016 \\
$144 \mathrm{~mm}$ & 105 & 131 & 157 & 183 & $1991-1995$ & 0.048 & 0.033 & 0.046 & 0.087 & 0.053 \\
$116 \mathrm{~mm}$ & 80 & 104 & 128 & 152 & $1996-2000$ & 0.036 & 0.030 & 0.026 & 0.042 & 0.034 \\
$122 \mathrm{~mm}$ & 113 & 119 & 125 & 131 & $2001-2005$ & 0.024 & 0.026 & 0.042 & 0.036 & 0.032 \\
$157 \mathrm{~mm}$ & 81 & 131 & 181 & 231 & $2006-2010$ & 0.031 & 0.022 & 0.037 & 0.032 & 0.031 \\
\hline
\end{tabular}




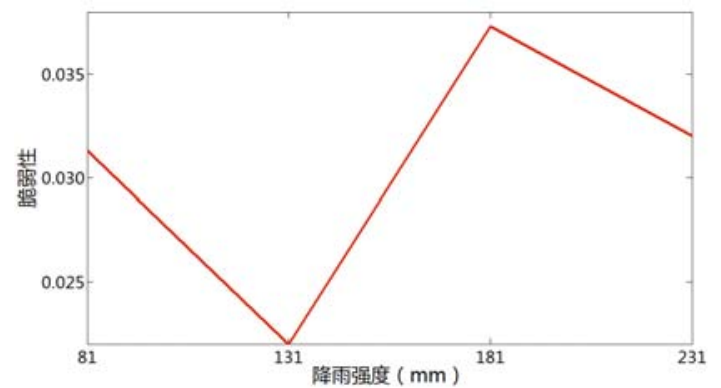

图 13 “十一五”期间汕头市洪涝灾害人口脆弱性曲线

\section{2 .3 时间轴上的人口脆弱性动态分析}

为了更清晰地表达人口脆弱性和降雨强度之 间的关系，以及脆弱性随时间推移的变化规律，将 5 个不同时期的人口脆弱性曲线合制成为三维立体图 （见图 14）。

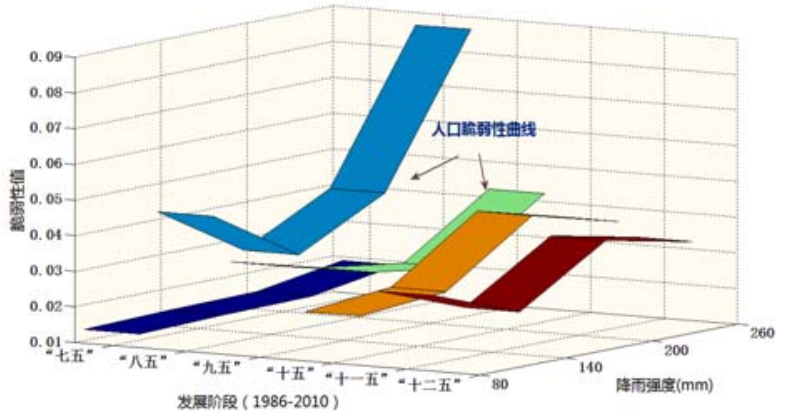

图 14 汕头市洪涝灾害的人口脆弱性动态变化曲线

从图 14 中, 我们可以看出每条脆弱性曲线都是 由 4 个离散点连接而成的, 但控制这些曲线变化的 降雨强度区间值是不一样的。

由表 8 可知，“七五”（1986-1990）期间的脆弱 性值变化范围是从 0.014 到 0.019 , 其对应的降雨强 度变化范围是从 $130 \mathrm{~mm}$ 到 $199 \mathrm{~mm}$; “八五” 期间的脆 弱性值范围是从 0.033 到 0.087 , 其对应的降雨强度 范围是 $105 \mathrm{~mm}$ 到 $183 \mathrm{~mm}$; “九五” 期间的脆弱性值范 围是从 0.026 到 0.042 , 其对应的降雨强度范围是 $80 \mathrm{~mm}$ 到 $152 \mathrm{~mm}$; “十五”期间的脆弱性值范围是从 0.024 到 0.042 , 其对应的降雨强度范围是 $113 \mathrm{~mm}$ 到 $131 \mathrm{~mm}$; “十一五” 期间的脆弱性值范围则是从 0.022 到 0.037 , 其对应的降雨强度范围是从 $81 \mathrm{~mm}$ 到 231mm。

如果我们分别求出汕头市“七五”、“八五”、“九 五”、“十五”和“十一五”期间的降雨强度均值和脆弱 性均值, 则可以得到均值意义上的随时间变化的人 口脆弱性动态变化曲线。因为 5 个发展时期的降雨 均值均大于 $100 \mathrm{~mm}$ 小于 $250 \mathrm{~mm}$, 属于大暴雨范围, 因此我们称此时的脆弱性曲线是大暴雨情景下的人 口脆弱性变化曲线 (见图 15)。

图 15 显示了从 1986-2010 期间汕头市暴雨洪涝 灾害人口脆弱性的变化趋势是先上升后减小，然后 趋于平缓。

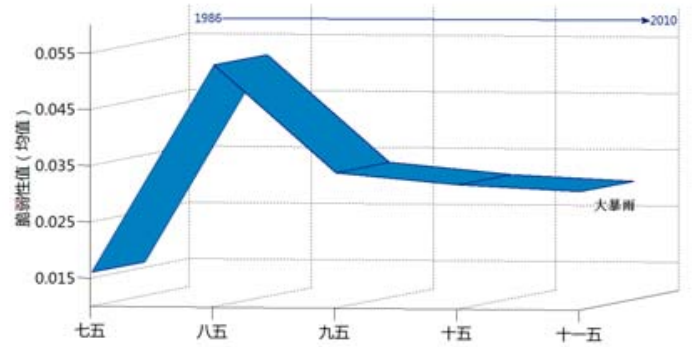

图 15 大暴雨 (均值) 条件下的汕头市洪涝灾害人口 脆弱性动态变化曲线

基于表 8 , 也可以找出各个时期降雨强度相近

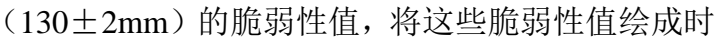
间轴上的人口脆弱性动态变化曲线后 (见图 16), 可 以看到在 $130 \mathrm{~mm}$ 左右降雨强度条件下的人口脆弱性 曲线变化趋势与大暴雨均值意义下的人口脆弱性变 化趋势稍有不同, 即从“七五”到“九五”, 人口脆弱性 变化的趋势都是先升后降，但到了“十五”和“十一 五”, 均值意义上的脆弱性曲线基本趋于平滑, 变化 幅度不大, 而 $130 \mathrm{~mm}$ 降雨条件下的脆弱性曲线却是 从“九五”到“十五”升高, 然后从“十五”到“十一五” 降低。

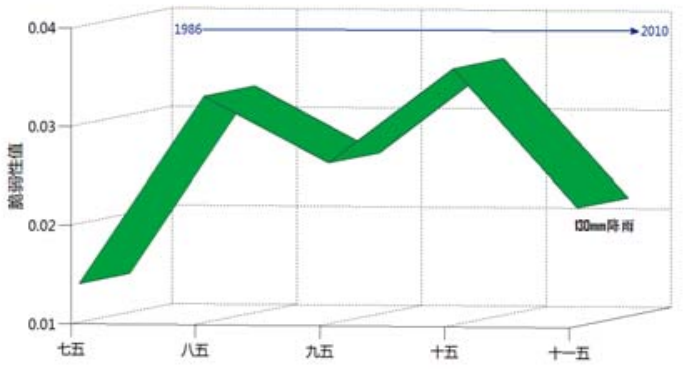

图 16 定量降雨 (130mm) 条件下的汕头市洪涝灾害 人口脆弱性动态变化曲线

\section{4 结论与讨论}

从1986-2010期间的多条脆弱性曲线的变化上 可以看出: 汕头市洪涝灾害的人口脆弱性并不是随 着经济的发展而持续降低或持续升高; 也不是人口 多了脆弱性就大, 人口少了脆弱性就小; 更不是降 雨量越大脆弱性就越大。同时, 致灾因子强度均值 意义上的脆弱性动态变化曲线和固定强度值对应的 脆弱性动态变化曲线也存在差异。在科学计算的前 提下, 脆弱性研究结果应该多样化, 以供决策者在 决策分析时可以根据自身需要多一些选择。

在没有主观赋分的前提下，利用信息扩散技术 和小样本数据分析得出的上述结果, 一方面说明人 口脆弱性的发展变化不只是单纯随着时间的变化、 人口的增长和致灾因子强度的大小而有规律的变化 的。另一方面说明承灾体脆弱性的变化受诸多因素 影响（如: 居民地的易损性、人口结构、人员流动、 
人口分布等), 致灾因子的变化并不能控制脆弱性的 变化。由于目前尚没有出现验证灾害风险评估和脆 弱性分析结果有效性的科学手段, 本文只能从理论 角度展开研究, 希望能够为自然灾害的脆弱性评估 研究提供一种新的思路。

注: 脆弱性和风险是两个不同的概念, 自然灾 害风险分析过程是致灾因子、承灾体和孕灾环境等 多因素综合分析的系统工程, 本文仅仅针对城市暴 雨洪涝灾害中的人口这一单一承灾体进行了脆弱性 分析研究, 而非系统性的风险分析。

\section{参考文献}

[1] Allianz. Allianz Risk Pulse-Focus: Floods[R]. 2011, 1-6

[2] Wilhelmi O V, Wilhite D A. Assessing Vulnerability to Agricultural Drought: A Nebraska Case Study[J]. Natural Hazards, 2002, 25: 37-58

[3] Dow K. Exploring Differences in Our Common Futures: the Meaning of Vulnerability to Global Environmental Change[J]. Geoforum, 1992, 23: 417-436

[4]Gilberto C Gallopin. Linkages between Vulnerability, Resilience and Adaptive Capacity. Global Environmental Change, 2006, 16: 293-303

[5]石勇. 灾害情景下城市脆弱性评估研究[D]. 博 士学位论文, 上海: 华东师范大学, 2010

Yong Shi. Research on Vulnerability Assessment of Cities on the Disaster Scenario[D]. Doctoral Dissertation, Shanghai: East China Normal University, 2010

[6] 张灵,陈晓宏, 王兆礼. 防洪系统脆弱性的模糊 极大熵诊断[J]. 系统工程理论与实践, 2011, 31 (8): 1600-1606

Zhang Ling, Chen Xiaohong, Wang Zhaoli. Fuzzy Diagnosis of Vulnerability to Flood Based on Maximum Entropy[J]. Systems Engineering Theory \& Practice, 2011, 31(8): 1600-1606

[7]史培军. 四论灾害系统研究的理论与实践 [J]. 自然灾害学报, 2005, 14(6): 1-7

Shi Peijun. Theory and Practice on Disaster System Research in A Fourth Time[J]. Journal of Natural Disasters, 2005, 14(6): 1-7

[8] Kröger W. Critical Infrastructures at Risk: A Need for A New Conceptual Approach and Extended Analytical Tools[J]. Reliability Engineering \& System Safety, 2008, 93(12): 1781-1787

[9] Kellenberga D. K. and Mobarakb A.M. Does Rising Income Increase or Decrease Damage Risk from Natural Disasters[J]. Journal of Urban Economics, 2008, 63(3): 788-802

[10] 陈颗,彭文涛,徐文立. 21 世纪地震灾害的一些 新特点 [J]. 地球科学进展, 2004, 19(3): 359-363

Chen Yong, Peng Wentao, Xu Wenli. New Feature of Earthquake Disaster in $21^{\text {st }}$ Century[J]. Advance in Earth Sciences, 2004, 19(3): 359-363

[11] 刘吉夫,陈颗,史培军等. 中国大陆地震风险分 析模型研究( II ): 生命易损性模型 [J]. 北京师 范大学学报(自然科学版), 2009, 45(4): 404-407.
Liu Jifu, Chen Yong, Shi Peijun et. al. Study on Seismic Risk Assessment in Mainland China (II): Life Vulnerability Model, Journal of Beijing Normal University (Natural Science), 2009, 45(4): 404-407

[12] 苏桂武,高庆华. 自然灾害风险的分析要素 [J]. 地学前缘, 2003, 10 (特刊): 272-279

$\mathrm{Su}$ Guiwu, Gao Qinghua. The Elements for Analyzing Natural Disaster Risk[J]. Earth Science Frontiers, 2003, 10 (SUPPL): 272-279

[13] 赵思健. 情景驱动的区域自然灾害风险分析 原理与应用[D]. 博士后出站报告, 2010, 北 京: 北京师范大学

Zhao Sijian. Scene-driven Risk Analysis to Regional Natural Disasters[D]. Postdocteral Research Report, 2010, Beijing: Beijing Normal University

[14] 黄崇福. 综合风险评估的一个基本模式 [J]. 应用基础与工程科学学报, 2008, 16(3): 371-381

Huang Chongfu. A Basic Model for Assessing Integrated Risk. Journal of Basic Science and Engineering, 2008, 16(3): 371-381

[15] 石勇,石纯,孙蕾等. 沿海城市自然灾害脆弱性 评价研究-以上海浦东新区为例 [J]. 中国人口. 资源与环境，2008，18(4)：24-27

Shi Yong, Shi Chun, Sun Lei et.al.. Vulnerability Assessment of Natural Disasters in Pudong District[J]. China Population, Resources and Environment, 2008, 18(4): 24-27

[16] 张斌,赵前胜,姜瑜君. 区域承灾体脆弱性指标 体系与精细量化模型研究 [J]. 灾害学, 2010, 25 (2): 36-40

[17] Zhang Bin, Zhao Qiansheng and Jiang Yujun. Research on Indexes System about Regional Vulnerability of Hazard-affected Bodies and Fine Spatial Quantitative Model[J]. Journal of Catastrophology, 2010, 25(2): 36-40

[18] 石勇,许世远,石纯等. 洪水灾害脆弱性研究进 展 $[\mathrm{J}]$. 地理科学进展, 2009, 28(1): 41-46

Shi Yong, Xu Shiyuan, Shi Chun et.al. A Review on Development of Vulneralbility Assessment of Floods[J]. Progress in Geography, 2009, 28(1): 41-46

[19] Huang C.F. Differences between Disaster Prediction and Risk Assessment in Natural Disasters [J]. Human and Ecological Risk Assessment, 2011, 17(3): 542-553

[20] Burton I, Kates R W, White G F. The Environment as Hazard[S]. Oxford Univ. Press, Oxford, 1978

[21] Covello V.T., Merkhoff er M.W. Risk Assessment Methods, Approaches for Assessing Health and Environmental Risks[M]. Plenum Press, New York, 1993

[22] Graham, W.J. A Procedure for Estimating Loss of Life Caused by Dam Failure[R]. Dam Safety Office Report, DSO-99-6, 1999

[23] Coburn A, Spence R. Earthquake Protection (2nd Edition) [M]. John Wiley \& Sons, 2002, Chichester

[24] Lind N., Hartford D. Probability of Human Instability in a Flooding: a Hydrodynamic Model[A], in: Melchers E and Stewart M.G. (ed) 
Proc. Of ICASP 8, Applications of Statistics and Probability[C], 2000, 1151-1156

[25] Jonkman S.N. Methode voor de bepaling van het aantal slachtoff ers ten gevolge van een grootschalige overstroming[R]. DWW report, 2004-042, 2004

[26] Finney D.J. Probit Analysis (3rd edition) [M]. London Cambridge University Press, 1972

[27] CPR Methods for the Determination of Possible Damage: People and Goods Through the Release of Hazardous Substances[M]. CPR, Voorburg, Netherlands, 1990

[28] Chongfu Huang, Claudio Moraga. Extracting Fuzzy If-then Rules by Using the Information Matrix Technique[J]. Journal of Computer and System Sciences, 2005，70: 26-52

[29] Chongfu Huang. Information Dissusion Techniques and Small-sample Problem[J]. International Journal of Information Technology \& Decision Making. 2002, 1(2): 229-249

[30] Huang Chongfu. Information Matrix and Application[J]. International Journal of General
Systems, 2001, 30(6): 603-622

[31] 黄崇福. 非完备样本知识的优化处理 [J]. 北 京师范大学学报(自然科学版), 1992, 28 (2): 129-135

Huang Chongfu. Optimality Processing to the Sample Knowledge of Non-completeness[J]. Journal of Beijing Normal University (Natural Science), 1992, 28(2): 129-135

[32] 王家鼎. 地理学研究中的模糊信息优化处理 方法 $[\mathrm{J}]$. 地理学与国土研究, 1999, 15(1): 75-80

Wang Jiading. Fuzzy Information Optimization Technology for Geography Research[J]. Geography and Territorial Research, 1999, 15(1): 75-80

[33] 黄崇福. 自然灾害风险评价: 理论与实践[M]. 北京: 科学出版社, 2005

Huang Chongfu. Risk Assessment of Natural Disaster: Theory \& Practice[M]. Beijing: Science Press, 2005 